\title{
SEM BSE 3D Image Analysis of Human Incus Bone Affected by Cholesteatoma Ascribes to Osteoclasts the Bone Erosion and VpSEM dEDX Analysis Reveals New Bone Formation
}

\author{
Michela Relucenti (D), ${ }^{1}$ Selenia Miglietta, ${ }^{1}$ Gabriele Bove, ${ }^{1}$ Orlando Donfrancesco, ${ }^{1}$ \\ Ezio Battaglione, ${ }^{1}$ Pietro Familiari, ${ }^{2}$ Claudio Barbaranelli, ${ }^{3}$ Edoardo Covelli, ${ }^{4}$ \\ Maurizio Barbara $₫{ }^{4}$ and Giuseppe Familiari ${ }^{1}$ \\ ${ }^{1}$ Department SAIMLAL Section of Human Anatomy, Laboratory of Electron Microscopy "Pietro M. Motta”, Sapienza University \\ of Rome, Via Alfonso Borelli 50, 00161 Rome, Italy \\ ${ }^{2}$ Department NESMOS, Neurosurgery Unit, Sapienza University of Rome, Via di Grottarossa 1039, 00189 Rome, Italy \\ ${ }^{3}$ Department of Psychology, Sapienza University of Rome, Via dei Marsi 78, 00185 Rome, Italy \\ ${ }^{4}$ Department NESMOS, ENT Unit, Sapienza University of Rome, Via di Grottarossa 1039, 00189 Rome, Italy
}

Correspondence should be addressed to Michela Relucenti; michela.relucenti@uniroma1.it

Received 29 July 2019; Accepted 22 January 2020; Published 17 February 2020

Guest Editor: Lavinia C. Ardelean

\begin{abstract}
Copyright (c) 2020 Michela Relucenti et al. This is an open access article distributed under the Creative Commons Attribution License, which permits unrestricted use, distribution, and reproduction in any medium, provided the original work is properly cited.
\end{abstract}

\begin{abstract}
Bone erosion is considered a typical characteristic of advanced or complicated cholesteatoma (CHO), although it is still a matter of debate if bone erosion is due to osteoclast action, being the specific literature controversial. The purpose of this study was to apply a novel scanning characterization approach, the BSE 3D image analysis, to study the pathological erosion on the surface of human incus bone involved by $\mathrm{CHO}$, in order to definitely assess the eventual osteoclastic resorptive action. To do this, a comparison of BSE 3D image of resorption lacunae (resorption pits) from osteoporotic human femur neck (indubitably of osteoclastic origin) with that of the incus was performed. Surface parameters (area, mean depth, and volume) were calculated by the software Hitachi MountainsMap@ from BSE 3D-reconstructed images; results were then statistically analyzed by SPSS statistical software. Our findings showed that no significant differences exist between the two groups. This quantitative approach implements the morphological characterization, allowing us to state that surface erosion of the incus is due to osteoclast action. Moreover, our observation and processing image workflow are the first in the literature showing the presence not only of bone erosion but also of matrix vesicles releasing their content on collagen bundles and self-immuring osteocytes, all markers of new bone formation on incus bone surface. On the basis of recent literature, it has been hypothesized that inflammatory environment induced by $\mathrm{CHO}$ may trigger the osteoclast activity, eliciting bone erosion. The observed new bone formation probably takes place at a slower rate in respect to the normal bone turnover, and the process is uncoupled (as recently demonstrated for several inflammatory diseases that promote bone loss) thus resulting in an overall bone loss. Novel scanning characterization approaches used in this study allowed for the first time the 3D imaging of incus bone erosion and its quantitative measurement, opening a new era of quantitative SEM morphology.
\end{abstract}

\section{Introduction}

Consensus-based recommendations for the definition of advanced or complicated cholesteatoma ( $\mathrm{CHO})$ [1] state that it is an agglomerate of keratinizing squamous epithelium, subepithelial connective tissue, that grows as a progressive accumulation of keratin debris with/without surrounding inflammatory reaction. Regarding its microstructure $\mathrm{CHO}$ is made of matrix (keratinized squamous epithelium), perimatrix (subepithelial connective tissue of variable thickness), and keratin debris. Bone erosion is considered a typical characteristic of cholesteatoma; however, it is still a matter of 
debate if bone erosion is due to osteoclast action, being present in literature conflicting results [2-7]. Scanning electron microscopy is an elective imaging technique for bone ultrastructural studies [8-12], so we observed by means of innovative SEM BSE 3D imaging and VPSEM EDX analysis that cholesteatoma affected incus bone surface, in order to accurately describe their surface modifications and finally assess if osteoclasts are directly responsible for bone resorption. To accomplish this task, we compared, using SEM BSE 3D imaging analyzed by Hitachi MountainsMap software, the fine structure of resorption pits observed on incus bone surface with the resorption lacunae from osteoporotic femur neck, indubitably of osteoclastic origin. Ultrastructural topography of incus bone surface was also studied through VPSEM EDX analysis.

\section{Materials and Methods}

2.1. Samples. We observed eighteen incus bones recovered during surgical procedures of $\mathrm{CHO}$ removal obtained with patients' informed consent and 1 unaffected incus bone (the control) from cadaver.

We studied eighteen femoral neck biopsies from postmenopausal women with hip arthrosis and osteoporosis who underwent surgical hip substitution, 1 femoral neck biopsy from woman without osteoporosis. BMD and Tscore to assess bone osteoporosis condition were evaluated by DEXA (Hologic Delphi) before the surgical operation. Samples were obtained with patients' informed consent.

The study was approved by the Institutional Ethics Board and adhered to the tenets of the Declaration of Helsinki.

\subsection{SEM Protocols}

2.2.1. Femoral Neck Biopsies. Samples were fixed immediately upon recovery in $2.5 \%$ glutaraldehyde in $\mathrm{PBS}$ at $4^{\circ} \mathrm{C}$ for $48 \mathrm{~h}$, then immersed in a $3 \%$ hydrogen peroxide solution for $48 \mathrm{~h}$ at room temperature (for bone marrow removal), and then rinsed with distilled water. Samples were then sonicated in a sonic device [13] in distilled water at room temperature, rinsed with distilled water, and dehydrated in acetone series. Samples were finally dried using a critical point dryer (Emitech K850, Emitech, Corato, Italy), mounted on aluminum stubs, platinum coated using an Emitech K 550 sputter coater (Emitech, Corato, Italy), and observed by a Hitachi FE SEM S 4000 operating at $7 \mathrm{kV}$. SEM micrographs were acquired with a DISS5 Digital Image Scanning System (point electronic, Germany).

2.2.2. Incus Preparation Protocol for SEM. Samples were fixed immediately upon recovery in $2.5 \%$ glutaraldehyde in PBS at $4^{\circ} \mathrm{C}$ for $48 \mathrm{~h}$; then, they were gently sonicated in an ultrasonic device (to remove excess of keratinizing squamous epithelium that would have prevented surface observation). Fifteen samples were prepared for SEM (as previously described for femur neck) and sputter coated with platinum using an Emitech K 550 sputter coater (Emitech, Corato, Italy). Observations were conducted by a Hitachi FE SEM S 4000 operating at $7 \mathrm{kV}$ and by a Hitachi SU 3500 (Hitachi High-
Technologies Europe GmbH, Mannheim, Germany), at $10 \mathrm{kV}$ in SE mode.

2.2.3. Incus Preparation Protocol for VPSEM and EDX Microanalysis. Three samples, after fixation in $2.5 \%$ glutaraldehyde in PBS at $4^{\circ} \mathrm{C}$ for $48 \mathrm{~h}$, were only gently sonicated in a sonic device [13] and then directly observed by a Hitachi SU 3500 (Hitachi High-Technologies Europe GmbH, Mannheim, Germany), operating at $5 \mathrm{kV}$ and $60 \mathrm{~Pa}$, in $\mathrm{BSE}$ COMPO mode without metal coating.

2.3. BSE 3D Image Analysis. Hitachi SU 3500 is equipped with a four-quadrant BSE detector that allows to acquire four images simultaneously with only one scan. The four pictures are then integrated into $3 \mathrm{D}$ images and processed to extract quantitative information (all those steps were performed by the software Hitachi Map 3D 7.4 Digital surf, Besançon, France). To obtain this kind of data is extremely useful to implement the morphological classification parameters usually used to characterize resorbing and forming bone surfaces. In fact, acquisition of quantitative resorption pit information such as area, mean depth, and volume allows to compare pits from different sources (femur and incus) and finally assess if they have the same origin. Regions containing resorption bay were analyzed in both incus bone and femur neck samples. BSE 3D images of well delimited resorption pits were acquired, 4 images were combined by the software, and 3D reconstruction was obtained. Resorption pit area, mean depth, and volume were extracted by MountainsMap software after 3D image reconstruction. In more detail, we performed single pit selection on the $3 \mathrm{D}$ image reconstruction, followed by automatic measurement of area, mean depth, and volume. Data were collected and statistically analyzed by SPSS statistical software. The following test was performed: summary statistic to assess the normality of distribution of pit area, mean depth, and volume values; independent sample $t$-test (assuming unequal and equal variances) was used to compare pits area, mean depth, and volume values between incus and femur samples.

2.4. EDX Microanalysis. The variable pressure scanning electron microscopy used in this study (VP-SEM, Hitachi SU3500) is equipped with dual energy dispersive X-ray spectroscopy (dEDS, Bruker XFlash ${ }^{\circledR}$ 6|60) detectors. This instrument has the ability to perform simultaneously multimodal imaging and spatial distribution chemical mapping, a truly powerful analytical approach to study biological surfaces in their native state. The XFlash ${ }^{\circledR} 6 \mid 60$ is particularly suitable for applications with relatively low X-ray yield, as common in the area of nanoanalysis.

2.5. Morphological Classification Parameters for Bone Surface Evaluation. Incus bone areas were classified as resorptive and forming bone surfaces, according to widely accepted morphological criteria described in literature [812, 14-17]. Briefly, resorbing bone surfaces are characterized by the presence of large resorption bay or scattered resorption pits (Howship's lacunae). Those structures observed by SEM show shining bright rounded edges, a floor made of partially demineralized collagen bundles and punctuated by 
narrow gutters, that appear darker at BSE imaging mode. Bone forming surfaces are characterized by an irregular surface, with collagen bundles undergoing mineralization, mineralizing matrix vesicles and shallow pits (the osteocytic lacunae) in which osteoblast/osteocyte immure themselves. They have an irregular ellipsoidal shape with a large range of variation [18].

\section{Results and Discussion}

Each CHO incus sample was observed by SEM at low magnification following a precise scanning pathway, in order to assess the general bone morphology and define areas suitable for high magnification observations. This method allowed counting of nutrient foramina opening onto the surface (49 foramina on 18 bones) and identification of areas with marked bone erosion and, interestingly, areas with new bone formation. It is still a matter of debate if bone erosion is due to osteoclast action; moreover, new bone formation was never been described in the incus bone affected by CHO. To get an insight on these findings, we performed observations at magnifications ranging from $400 x$ to $600 x, 3 D$ image reconstruction, and EDS analysis.

3.1. Observations of Normal Sample Surface. Before showing images of samples with resorption areas, two images of normal surfaces are presented (Figure 1): normal incus bone surface (Figure 1(a)) and normal trabecular bone (Figure 1(b)). The surface of both bones is devoid of resorption bays.

3.2. Observations of Resorbing Areas. Images of $\mathrm{CHO}$ incus bone surface showed $67 \%$ of nutritive foramina surrounded by large resorption bays that seem to radiate from nutritive foramen opening (Figures 2(a) and 2(b)).

Observed at higher magnification $\mathrm{CHO}$ incus bone resorption bays and pits (Figure $3(\mathrm{a})$ ) resemble in all respects those on the surface of femur neck with osteoporosis (Figure 3(b)).

To definitely assess if incus bone resorption bay is a product of osteoclasts action, we used Hitachi MountainsMap ( software to perform a 3D reconstruction from 4 BSE mode images (Figure 4).

A small area was extracted from a 3D-reconstructed image, and each single pit in the small area was analyzed by the software to calculate: area, mean depth, and volume (Figures 5(a) and 5(b)).

We analyzed 79 pits, for each considered parameter values which were recorded and statistically evaluated by SPSS statistical software. Firstly, a summary statistic was performed on data collected for each parameter, to assess normality of distribution (Table 1). For all values, data distribution was normal, so $t$-test was performed between values of each parameter measured on incus and femur to assess difference between values (Table 2, Figure 6). Two series of independent sample $t$-test, assuming, respectively, unequal and equal variances, were conducted to compare area, mean depth, and volume values between incus and femur. Both series evidenced that no difference exists between the two groups $(p>0.05)$ for each considered parameter.
3.3. Observation of New Bone-Forming Area. The detailed incus surface observation allowed another interesting finding, the observation of new bone-forming areas on incus surface. Our SEM images are the first to show this process on incus. Mineralizing vesicles releasing their content on collagen bundles are shown in Figures 7(a) and 7(b). SE mode allows the detailed visualization of collagen fibre meshwork while BSE mode clearly points out that matrix vesicles are filled with a high molecular weight content and that collagen bundles have different mineralization degree (lighter or darker areas).

These areas were also analyzed in uncoated samples by variable pressure SEM dEDS analysis. Variable pressure SEM allows the observation of uncoated samples, avoiding metal coating disturbance during elemental analysis. Areas containing calcified matrix vesicles (Figure 8(a)) were analyzed by dEDS. Elemental mapping (Figure 8(b)) clearly shows the presence of calcium in matrix vesicles, while sulphur, contained in matrix proteoglycans, is present only in the surrounding extracellular matrix. Calcium and phosphorus are the characteristic elements of bioapatite [19-21]. The elemental mapping clearly demonstrates that matrix vesicles have a calcium phosphate content.

A later stage in new bone formation is represented by osteocyte self-immuring in forming bone areas. In Figure 9, detailed images of osteocytic lacunae are presented for the first time in which the osteocyte self immure on incus surface. Here again, they are perfectly superimposable with osteocytic lacunae on femur neck samples. Osteocytic lacunae appear surrounded by fully mineralized collagen bundles. At higher magnification (Figure $8(\mathrm{~b})$ ), on the floor of the osteocytic lacuna, the not yet fully mineralized collagen fibres and the deep holes are visible in which osteocyte cellular processes nestle.

Prominent theories on bone resorption in $\mathrm{CHO}$ are osteoclast activation; pressure necrosis; and acid lysis, enzyme mediation, and inflammatory mediation [22]. The mechanism of bone erosion in middle ear cholesteatoma remains still unclear, although its histopathology has been intensively studied.

In some studies $[2,3]$, osteoclast was not observed in resorbing bone areas of incus with $\mathrm{CHO}$; in others [4-7], they were reported. This is probably due to the transient nature of osteoclasts; they have a relatively short life and, being surgical procedures done after inflammation control, they may be not present at time of sample recovery and fixation.

Our results showed that no difference exists between area, mean depth, and volume values between incus and femur resorption pit, allowing us to state that surface erosion on the incus is due to osteoclast action.

Osteoclasts are multinucleated cells, they differentiate from monocyte-lineage hematopoietic precursor cells [23]. Macrophage colony-stimulating factor (M-CSF) and receptor activator of nuclear factor $\kappa \mathrm{B}$ ligand (RANKL) regulate both differentiation and activation of osteoclasts [24]. In several inflammatory diseases, like rheumatoid arthritis, pathological bone loss is observed, together with RANKL overproduction $[25,26]$. Immune cells such as T-lymphocytes and macrophages that infiltrate into damaged areas are major sources of RANKL [27], and fibroblasts in the cholesteatoma 


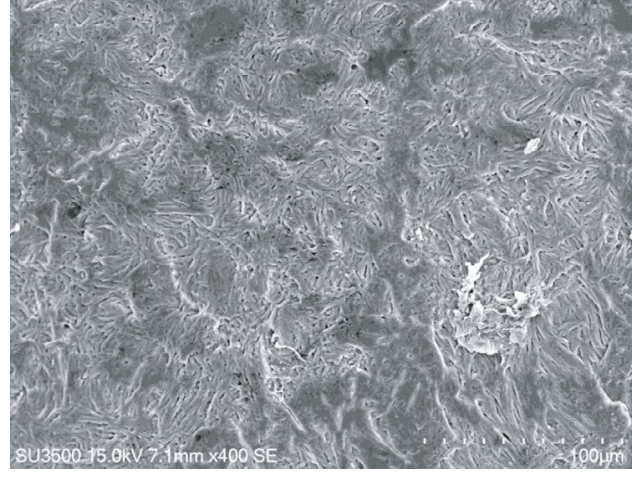

(a)

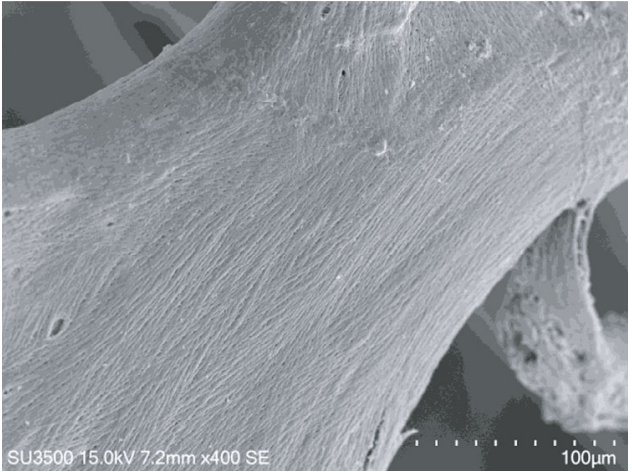

(b)

Figure 1: (a) SE mode, 400x. Incus bone surface from cadaver, normal surface. (b) SE mode, 400x. Trabecular bone from patient without osteoporosis, normal surface.

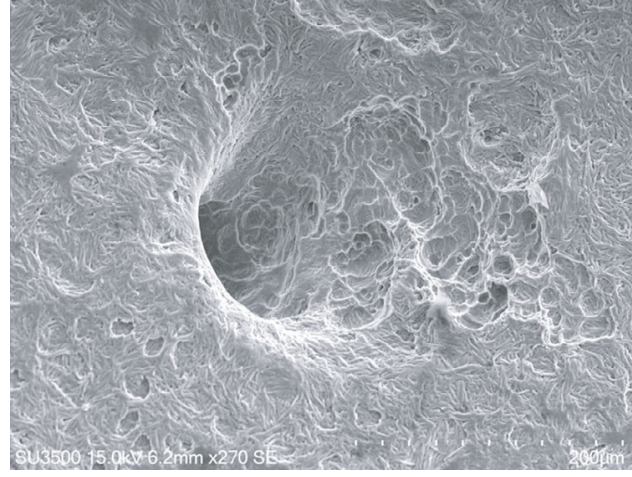

(a)

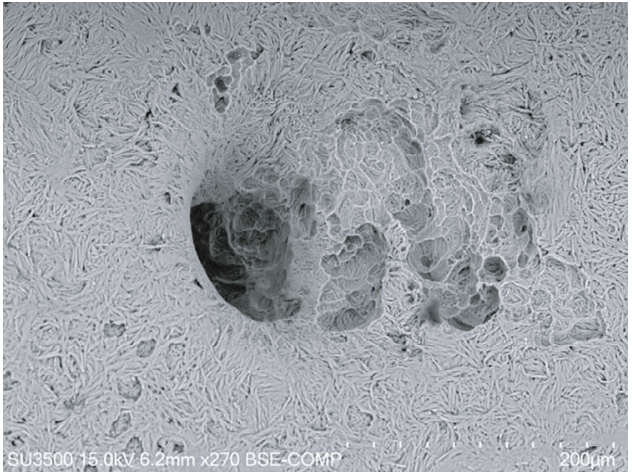

(b)

FIGURE 2: (a) SE mode, 270x. Nutritive foramen from CHO incus bone. On the right side of the image, large resorption bays, extending since into the foramen, are visible. On the left corner of the picture, osteocytic lacunae are visible. (b) BSE-COMP mode, 270x of same sample. Darker (demineralized) areas correspond to deeper resorption bays. This field shows both bone resorption and bone formation phenomena.

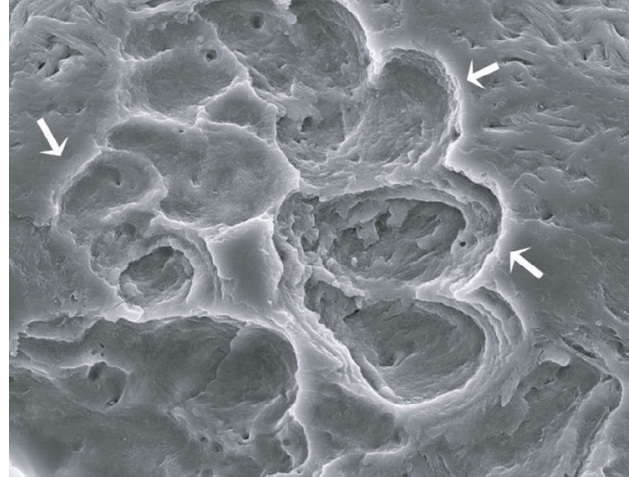

SE $20 \mathrm{kV}$ WD $18 \mathrm{~mm} 700 \mathrm{X}$ $\vdash 20 \mu \mathrm{m} \longrightarrow$

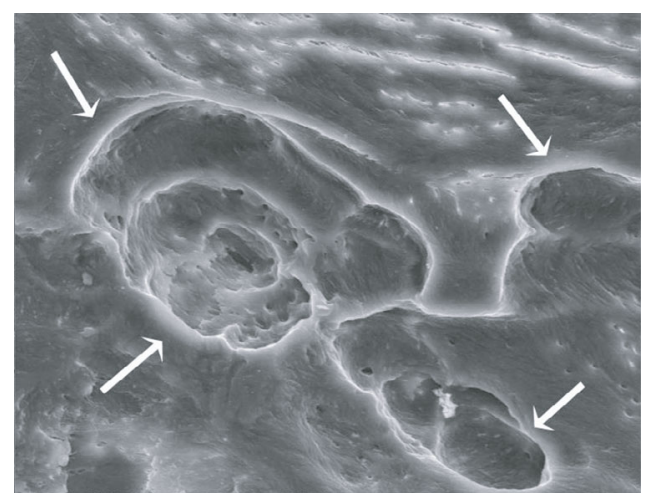

SE $20 \mathrm{Kv}$ WD $18 \mathrm{~mm} 600 \mathrm{X}$ $\vdash 20 \mu \mathrm{m} \longrightarrow$

(b)

FIGURE 3: (a) FE SEM 700x, CHO incus bone resorption bay at higher magnification, osteoclast snake trail pathway is visible (arrows). At the center of the resorption bay, a small promontory rises being relatively resistant to resorption. (b) FE SEM, 600x, osteoclastic resorption bay on osteoporotic human femur neck (arrows), they are unequivocally of osteoclast origin and are undistinguishable from those in (a).

perimatrix express RANKL [28]. The effector cell of focal osteolysis is the osteoclast, but cytokines are key regulators of inflammatory osteolysis [29]. IL-1, IL-6, TNF- $\alpha$, and prostaglandin E2 (PGE2) have been investigated as inflammatory mediators of cholesteatoma progression. They are assumed to enhance bone resorption by activating osteoclasts [3032], and inflammation has been confirmed to be essential for cholesteatoma formation, growth, and expansion, 


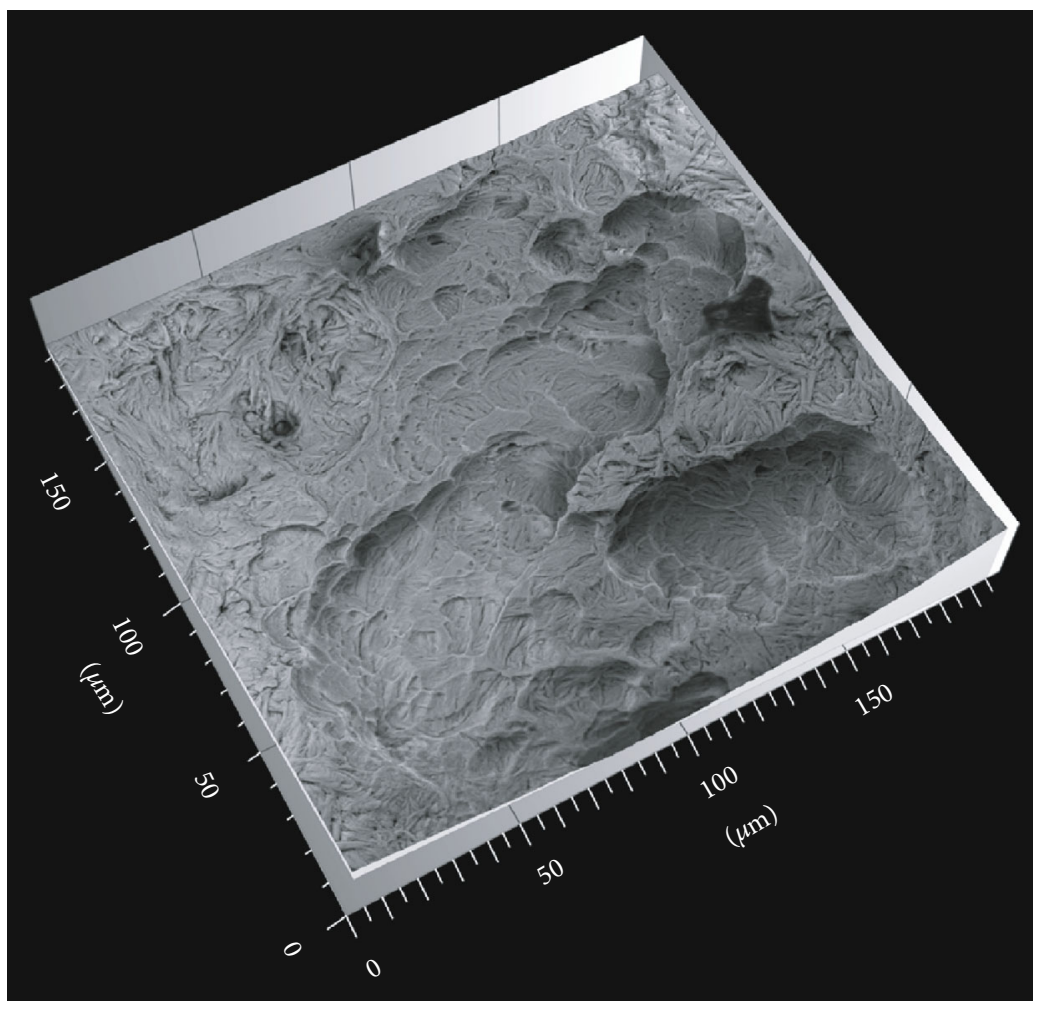

FIGURE 4: 3D reconstruction from 4 images in BSE mode. Each resorption bay contains several pits.
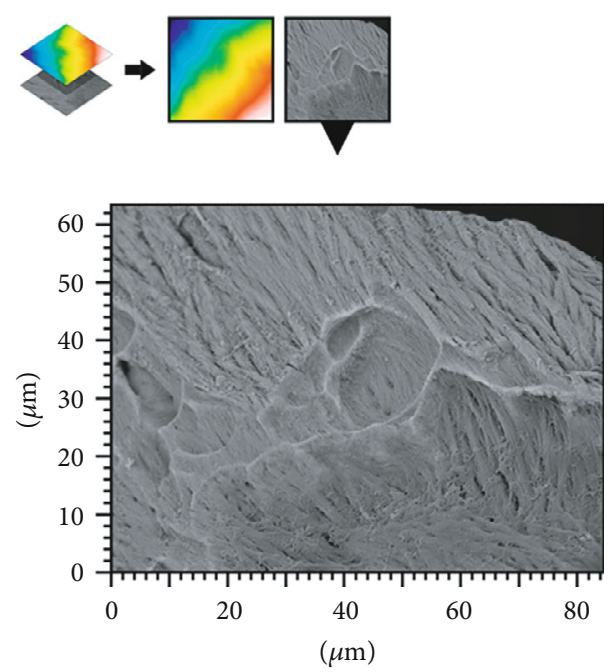

(a)

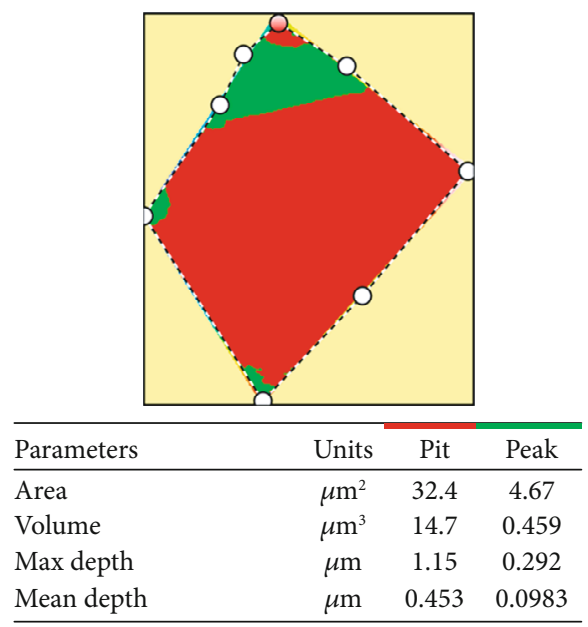

(b)

FIGURE 5: (a) The extracted area of a resorption bay from a larger 3D-reconstructed image. (b) A delimited single pit from which software calculated parameter values.

TABLE 1: Summary statistic of area, mean depth, and volume values.

\begin{tabular}{lcccc}
\hline Pit & Distribution & $\begin{array}{c}\text { Area } \mu \mathrm{m}^{2} \\
\text { Arithmetic mean } \pm \mathrm{ds}\end{array}$ & $\begin{array}{c}\text { Mean depth } \mu \mathrm{m} \\
\text { Arithmetic mean } \pm \mathrm{ds}\end{array}$ & $\begin{array}{c}\text { Volume } \mu \mathrm{m}^{3} \\
\text { Arithmetic mean } \pm \mathrm{ds}\end{array}$ \\
\hline Incus & Normal & $120.48 \pm 8.54$ & $0.799 \pm 0.10$ & $96.48 \pm 13.16$ \\
Femur neck & Normal & $121.34 \pm 23.2047$ & $0.784 \pm 0.16$ & $94.99 \pm 23.65$ \\
\hline
\end{tabular}


TABLE 2: Independent sample $t$-test on area, mean depth, and volume values.

\begin{tabular}{|c|c|c|c|c|c|c|}
\hline & \multicolumn{2}{|c|}{ Area } & \multicolumn{2}{|c|}{ Mean depth } & \multicolumn{2}{|c|}{ Volume } \\
\hline & Incus & Femur & Incus & Femur & Incus & Femur \\
\hline Sample size & 79 & 79 & 79 & 79 & 79 & 79 \\
\hline Arithmetic mean & 120.48 & 121.34 & 0.799 & 0.784 & 96.48 & 94.99 \\
\hline $95 \% \mathrm{CI}$ for the mean & 118.57 to 122.39 & 116.15 to 126.54 & 0.77 to 0.82 & 0.74 to 0.82 & 93.51 to 99.45 & 89.69 to 100.28 \\
\hline Variance & 72.95 & 538.45 & 0.011 & 0.025 & 173.31 & 559.32 \\
\hline St deviation & 8.54 & 23.20 & 0.10 & 0.16 & 13.16 & 23.65 \\
\hline St error mean & 0.96 & 2.61 & 0.011 & 0.018 & 1.49 & 2.66 \\
\hline$F$-test equal variances & \multicolumn{2}{|c|}{$p<0.001$} & \multicolumn{2}{|c|}{$p<0.001$} & \multicolumn{2}{|c|}{$p<0.001$} \\
\hline$t$-test equal variances & \multicolumn{2}{|c|}{$\begin{array}{c}t(156)=0.310 \\
p=0.7568\end{array}$} & \multicolumn{2}{|c|}{$\begin{array}{c}t(155)=-0.688 \\
p=0.4922\end{array}$} & \multicolumn{2}{|c|}{$\begin{array}{c}t(156)=-0.489 \\
p=0.6258\end{array}$} \\
\hline Levene $t$-test unequal variances & \multicolumn{2}{|c|}{$\begin{array}{c}t(98.76)=0.310 \\
p=0.7570\end{array}$} & \multicolumn{2}{|c|}{$\begin{array}{c}t(122.43)=-0.688 \\
p=0.4924\end{array}$} & \multicolumn{2}{|c|}{$\begin{array}{c}t(134.18)=-0.489 \\
p=0.6249\end{array}$} \\
\hline
\end{tabular}
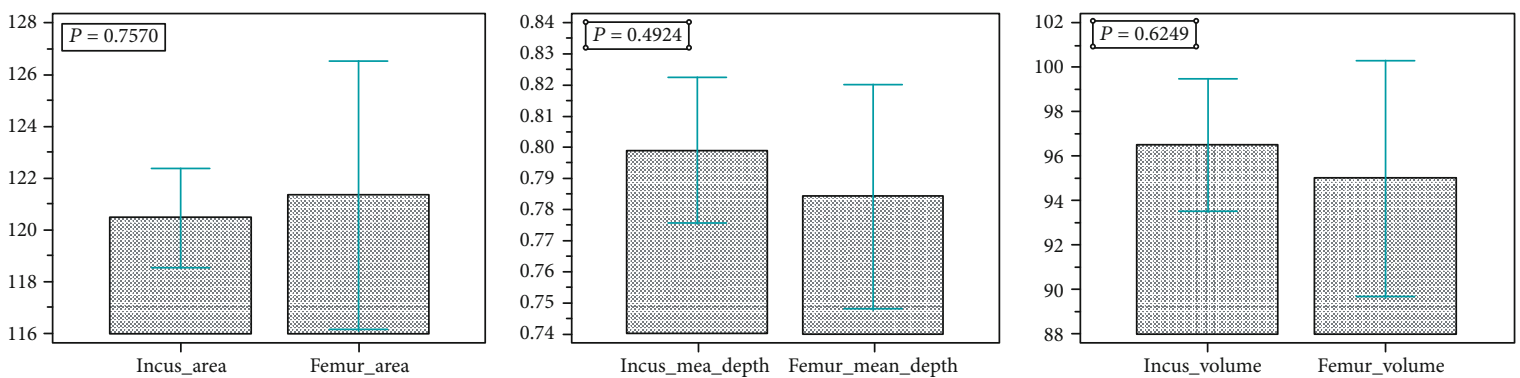

FIGURE 6: Graphs represent distribution of pit measurement data (from the left to the right): incus area vs. femur area; incus mean depth vs. femur mean depth; incus volume vs. femur volume.

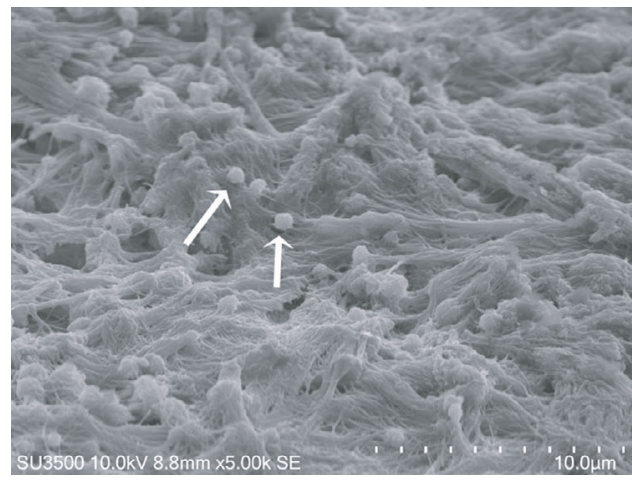

(a)

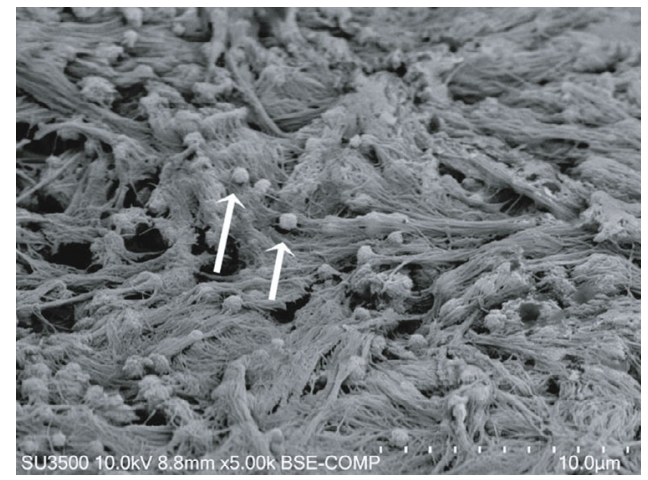

(b)

FIGURE 7: SE, BSE comp 5000x, new bone formation on CHO incus bone surface. (a) SE mineralizing matrix vesicles releasing their content on collagen bundles (arrows). (b) BSE comp mineralizing matrix vesicles (arrows) appear as bright and rough spheres. Collagen fibres and bundles with variable mineralization degree are visible. Mineralized areas appear lighter at BSE mode.

including the bone resorption process $[22,33,34]$. Inflammatory cells were observed in our samples; in Figure 10, a rare coexistence of a macrophage (blue), a lymphocyte (red), and an osteoclast is presented [35-37].

Bone homeostasis is maintained balancing boneresorbing osteoclast and bone-forming osteoblast activity, alteration of this balance causes bone loss, that is not recov- ered by new bone formation. In fact, in inflammation, disease-like RA bone erosion results from excessive bone resorption and markedly limited bone formation [38]. We observed typical morphological markers of new bone formation on incus by $\mathrm{CHO}$, but this phenomenon probably happens at a slower rate than bone resorption, so that bone loss is not compensed. 


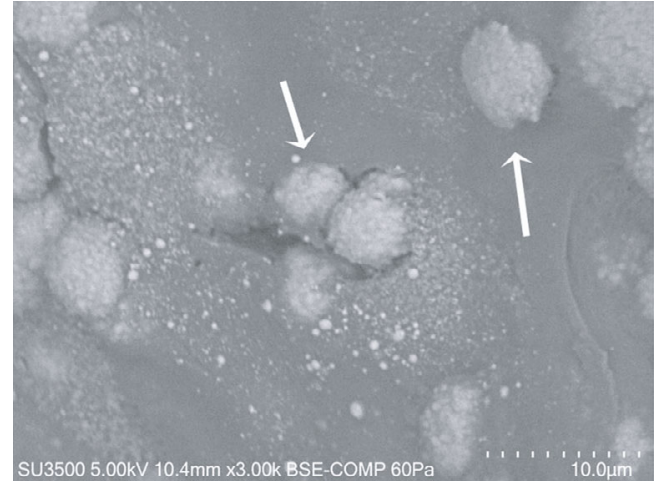

(a)

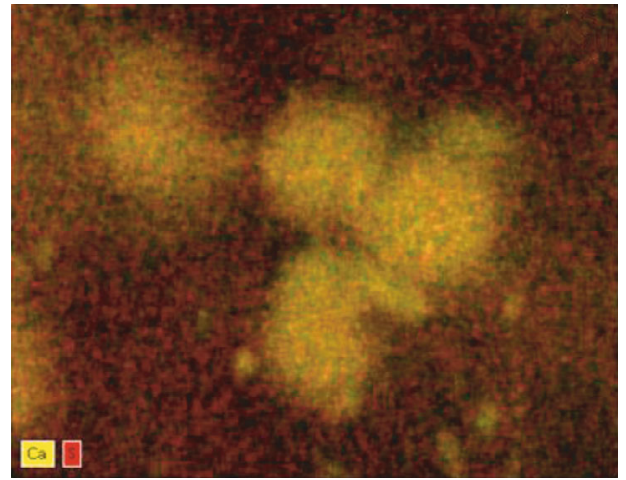

(b)

FIGURE 8: BSE Comp, 3000x, dEDS analysis, confirmation of new bone formation on CHO incus bone. (a) VP SEM BSE image shows matrix vesicles (arrows). (b) Elemental distribution (dEDS analysis) allows identification of chemical species, calcium in matrix vesicles (yellow) and sulphur in extracellular matrix (red).

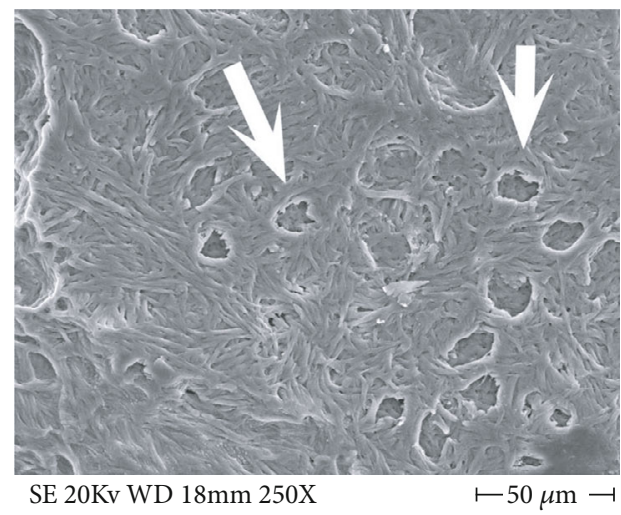

(a)

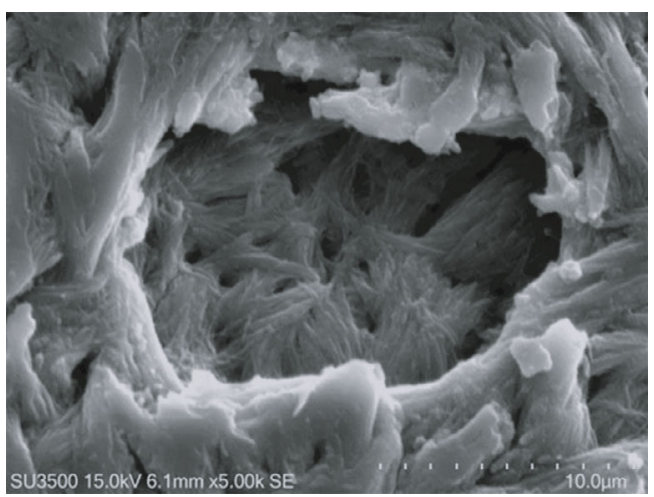

(b)

Figure 9: New bone formation on CHO incus bone surface (a), FE SEM, 250x, osteocytic lacunae (arrows) formed by self-immuring osteocytes. (b) SE, 5000x, high magnification of an osteocytic lacuna, the floor appears less mineralized and spotted by deep holes to accommodate osteocyte cellular processes.

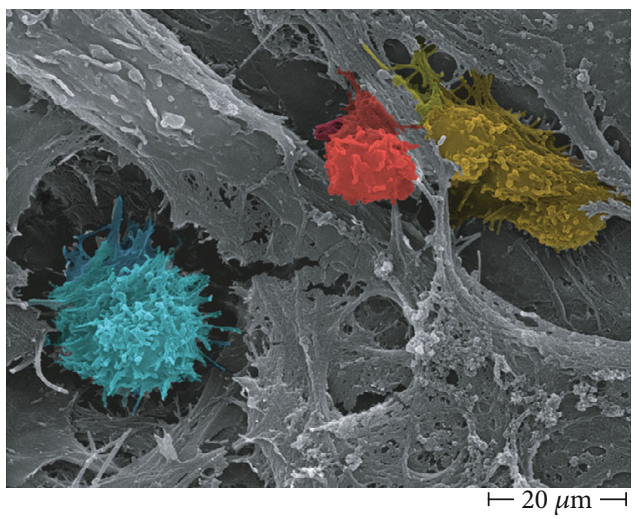

FIGURE 10: Inflammatory cells and an osteoclast on incus affected by CHO surface, FE SEM 3000x. Active macrophage (blue), lymphocyte (red), and osteoclast (yellow).

\section{Conclusions}

The innovative quantitative approach used in this paper implements the classical surface morphological characteriza- tion, allowing us to state that surface erosion of the incus is due to osteoclast action. Moreover, our observation and processing image workflow are the first in the literature showing the presence not only of bone erosion but also of matrix vesicles releasing their content on collagen bundles and selfimmuring osteocytes, all markers of new bone formation on incus bone surface. On the basis of recent literature [2234], it has been hypothesized that inflammatory environment induced by $\mathrm{CHO}$ may trigger the osteoclast activity, eliciting bone erosion; we can provide a morphological evidence of this hypothesis in Figure 9; in fact, a T-lymphocyte, a macrophage, and an osteoclast were photographed one near the other; the photograph gives the impression of witnessing the paracrine molecular dialogue between these cells [2234]. The observed new bone formation probably takes place at a slower rate in respect to the normal bone turnover, and the process is uncoupled (as recently demonstrated for several inflammatory diseases that promote bone loss) thus resulting in an overall bone loss. Novel scanning characterization approaches used in this study allowed for the first time the $3 \mathrm{D}$ imaging of incus bone erosion and its quantitative measurement, opening a new era of biological quantitative 
SEM morphology. Taken all together, our morphological results let us hypothesize that cholesteatoma creates an environment of chronic infection with peculiar biochemical characteristics that alters normal bone turnover on incus bone. Targeting the cell population of the inflammatory microenvironment (which produce molecules that stimulate osteoclast activity) will open new therapeutic options, in particular in the field of noninvasive therapies, allowing to inhibit bone erosion development in the acquired middle ear cholesteatoma.

\section{Data Availability}

Data are stored in computer of our institution and are available upon request.

\section{Conflicts of Interest}

The authors declare that they have no conflicts of interest.

\section{Acknowledgments}

This study was funded by the Sapienza University of Rome Research projects funding.

\section{References}

[1] E. Olszewska, J. Rutkowska, and N. Özgirgin, "Consensusbased recommendations on the definition and classification of cholesteatoma," The Journal of International Advanced Otology, vol. 11, no. 1, pp. 81-87, 2015.

[2] H. Koizumi, H. Suzuki, R. Kawaguchi et al., "Presence of osteoclasts in middle ear cholesteatoma: a study of undecalcified bone sections," Acta Oto-Laryngologica, vol. 137, no. 2, pp. 127-130, 2017.

[3] H. Koizumi, H. Suzuki, S. Ikezaki, T. Ohbuchi, K. Hashida, and A. Sakai, "Osteoclasts are not activated in middle ear cholesteatoma," Journal of Bone and Mineral Metabolism, vol. 34, no. 2, pp. 193-200, 2016.

[4] R. A. Chole, "Cellular and subcellular events of bone resorption in human and experimental cholesteatoma: the role of osteoclasts," Laryngoscope, vol. 94, no. 1, pp. 76-95, 1984.

[5] R. A. Chole, "Osteoclasts in chronic otitis media, cholesteatoma, and otosclerosis," The Annals of Otology, Rhinology, and Laryngology, vol. 97, no. 6, pp. 661-666, 1988.

[6] R. Imai, T. Sato, Y. Iwamoto et al., "Osteoclasts modulate bone erosion in cholesteatoma via RANKL signaling," Journal of the Association for Research in Otolaryngology, vol. 20, no. 5, pp. 449-459, 2019.

[7] Y. Iwamoto, K. Nishikawa, R. Imai et al., "Intercellular communication between keratinocytes and fibroblasts induces local osteoclast differentiation: a mechanism underlying cholesteatoma-induced bone destruction," Molecular and Cellular Biology, vol. 36, no. 11, pp. 1610-1620, 2016.

[8] A. Boyde, "Scanning electron microscopy of bone," in Bone Research Protocols, A. I. Idris, Ed., vol. 1914 of Methods in Molecular Biology, Humana Press, New York, NY, 2019.

[9] A. Boyde and S. J. Jones, "Scanning electron microscopy of bone: instrument, specimen, and issues," Microscopy Research and Technique, vol. 33, no. 2, pp. 92-120, 1996.
[10] S. J. Jones, A. Boyde, N. N. Ali, and E. Maconnachie, "A review of bone cell and substratum interactions: an illustration of the role of scanning electron microscopy," Scanning, vol. 7, no. 1, p. 24, 1985.

[11] A. Boyde and M. H. Hobdell, "Scanning electron microscopy of lamellar bone," Zeitschrift für Zellforschung und Mikroskopische Anatomie, vol. 93, no. 2, pp. 213-231, 1968.

[12] M. Relucenti, S. Miglietta, E. Covelli et al., "Ciliated cell observation by SEM on the surface of human incudo-malleolar-joint articular cartilage: are they a new chondrocyte phenotype?," Acta Oto-Laryngologica, vol. 139, no. 5, pp. 439-443, 2019.

[13] G. Familiari, A. Familiari, G. Macchiarelli, L. J. A. DiDio, and P. M. Motta, "The use of sonic frequencies as a clearing agent of specimens to be observed with scanning electron microscopy," Scanning Microscopy, vol. 7, no. 1, pp. 107-114, 1993.

[14] D. W. Dempster, E. Shane, W. Horbert, and R. Lindsay, "A simple method for correlative light and scanning electron microscopy of human iliac crest bone biopsies: qualitative observations in normal and osteoporotic subjects," Journal of Bone and Mineral Research, vol. 1, no. 1, pp. 15-21, 1986.

[15] S. Ren, H. Takano, and K. Abe, "Two types of bone resorption lacunae in the mouse parietal bones as revealed by scanning electron microscopy and histochemistry," Archives of Histology and Cytology, vol. 68, no. 2, pp. 103-113, 2005.

[16] H. Hashizume, K. Abe, and T. Ushiki, "Detection of mineral density on the surface of mouse parietal bones: backscattered electron imaging of low accelerating voltage scanning electron microscopy," Archives of Histology and Cytology, vol. 60, no. 2, pp. 195-204, 1997.

[17] R. J. Murrills, E. Shane, R. Lindsay, and D. W. Dempster, "Bone resorption by isolated human osteoclasts in vitro: effects of calcitonin," Journal of Bone and Mineral Research, vol. 4, no. 2, pp. 259-268, 1989.

[18] B. R. McCreadie, S. J. Hollister, M. B. Schaffler, and S. A. Goldstein, "Osteocyte lacuna size and shape in women with and without osteoporotic fracture," Journal of Biomechanics, vol. 37, no. 4, pp. 563-572, 2004.

[19] B. Alexander, T. L. Daulton, G. M. Genin et al., "The nanometre-scale physiology of bone: steric modelling and scanning transmission electron microscopy of collagenmineral structure," Journal of The Royal Society Interface, vol. 9, no. 73, pp. 1774-1786, 2012.

[20] N. Kourkoumelis, I. Balatsoukas, and M. Tzaphlidou, "Ca/P concentration ratio at different sites of normal and osteoporotic rabbit bones evaluated by Auger and energy dispersive X-ray spectroscopy," Journal of Biological Physics, vol. 38, no. 2, pp. 279-291, 2012.

[21] V. Cottignoli, M. Relucenti, G. Agrosì et al., "Biological niches within human calcified aortic valves: towards understanding of the pathological biomineralization process," BioMed Research International, vol. 2015, 542610 pages, 2015.

[22] S. Xie, X. Wang, J. Ren, and W. Liu, "The role of bone resorption in the etiopathogenesis of acquired middle ear cholesteatoma," European Archives of Oto-Rhino-Laryngology, vol. 274, no. 5, pp. 2071-2078, 2017.

[23] W. J. Boyle, W. S. Simonet, and D. L. Lacey, "Osteoclast differentiation and activation," Nature, vol. 423, no. 6937, pp. 337342, 2003.

[24] S. L. Teitelbaum and F. P. Ross, "Genetic regulation of osteoclast development and function," Nature Reviews. Genetics, vol. 4, no. 8, pp. 638-649, 2003. 
[25] K. W. Kim, M. L. Cho, S. H. Lee et al., "Human rheumatoid synovial fibroblasts promote osteoclastogenic activity by activating RANKL via TLR-2 and TLR-4 activation," Immunology Letters, vol. 110, no. 1, pp. 54-64, 2007.

[26] H. Takayanagi, H. Iizuka, T. Juji et al., "Involvement of receptor activator of nuclear factor kappaB ligand/osteoclast differentiation factor in osteoclastogenesis from synoviocytes in rheumatoid arthritis," Arthritis and Rheumatism, vol. 43, no. 2, pp. 259-269, 2000.

[27] I. B. Mcinnes and G. Schett, "The pathogenesis of rheumatoid arthritis," The New England Journal of Medicine, vol. 365, no. 23, pp. 2205-2219, 2011.

[28] G. Mbalaviele, D. V. Novack, G. Schett, and S. L. Teitelbaum, "Inflammatory osteolysis: a conspiracy against bone," The Journal of Clinical Investigation, vol. 127, no. 6, pp. 20302039, 2017.

[29] T. T. Jung and S. K. Juhn, "Prostaglandins in human cholesteatoma and granulation tissue," The American Journal of Otology, vol. 9, no. 3, pp. 197-200, 1988.

[30] J. Kuczkowski, M. Sakowicz-Burkiewicz, E. Iżycka-Świeszewska, B. Mikaszewski, and T. Pawełczyk, "Expression of tumor necrosis factor- $\alpha$, interleukin- $1 \alpha$, interleukin- 6 and interleukin- 10 in chronic otitis media with bone osteolysis," ORL, vol. 73, no. 2, pp. 93-99, 2011.

[31] R. F. Vitale and F. de Andrade Quintanilha Ribeiro, "The role of Tumor Necrosis Factor -Alpha (TNF- $\alpha$ ) in bone resorption present in middle ear cholesteatoma," Brazilian Journal of Otorhinolaryngology, vol. 73, no. 1, pp. 117-121, 2007.

[32] W. Likus, K. Siemianowicz, J. Markowski et al., "Bacterial infections and osteoclastogenesis regulators in men and women with cholesteatoma," Archivum Immunologiae et Therapiae Experimentalis, vol. 64, no. 3, pp. 241-247, 2016.

[33] F. A. W. Peek, M. A. Huisman, R. J. Berckmans, A. Sturk, J. van Loon, and J. J. Grote, "Lipopolysaccharide concentration and bone resorption in cholesteatoma," Otology \& Neurotology, vol. 24, no. 5, pp. 709-713, 2003.

[34] S. Yetiser, B. Satar, and N. Aydin, "Expression of epidermal growth factor, tumor necrosis factor-alpha, and interleukinlalpha in chronic otitis media with or without cholesteatoma," Otology \& Neurotology, vol. 23, no. 5, pp. 647-652, 2002.

[35] P. M. Motta, P. M. Andrews, and K. R. Porter, Eds., Microanatomy of cell and tissue surfaces. An atlas of scanning electron microscopy, Lea \& Febiger, Philadelphia, 1977.

[36] D. W. Fawcett, Ed., The Cell, W.B. Saunders, Philadelphia, 1981.

[37] P. M. Motta, Ed., "Cells and Tissues, A three-dimensional approach by modern techniques in microscopy," in Prog. Clin. Biol. Res., vol. 295, Alan R. Liss, Inc., New York, 1989.

[38] J. H. Shim, Z. Stavre, and E. M. Gravallese, "Bone loss in rheumatoid arthritis: basic mechanisms and clinical implications," Calcified Tissue International, vol. 102, no. 5, pp. 533-546, 2018. 